\title{
Blocked-D phenomenon in hemolytic disease of fetus and newborn with multiple maternal anti- rhesus antibodies
}

\begin{abstract}
Background: The blocking of D antigen sites of $\mathrm{RBC}$ membrane of the fetus by the passively transferred IgG anti-D in cases of Hemolytic Disease of fetus and new born (HDFN) is called blocked- D phenomenon. The coating of maternal IgG type of anti-D prevents the agglutination of the Rh-(D) antigen positive red blood cells (RBC) by the IgM D-antigen typing reagents. We are reporting two cases of Rh-(D) HDFN which were falsely typed as $\mathrm{Rh}(\mathrm{D})$ antigen negative with routine typing reagents and had multiple alloantibodies in the maternal serum.
\end{abstract}

Aims: To rule out HDFN and to confirm the Rh-(D) status of baby, to detect the presence of other allo-antibodies in the maternal serum that can complicate future transfusions in mother.

Materials: After routine blood grouping, sample of baby was subjected to adsorptionelution studies and maternal serum was used for antibody screening and identification

Results: In both the cases, blocked-D phenomenon got detected and there were multiple anti-rhesus antibodies other than anti-D in the maternal serum.

Conclusion: Antibody identification in antenatal women is important in the case management of HDFN to protect future pregnancies and to avoid the risk of mismatched transfusions.

Keywords: blocked-d phenomenon, HDFN, allo-antibodies, adsorption-elution, anti-D
Volume 7 Issue 2 - 2019

\section{Anila Mani, Poornima AP, Debasish Gupta \\ Department of Transfusion Medicine, Sree Chitra Tirunal Institute for Medical Sciences and Technology, India}

Correspondence: Dr. Debasish Gupta, Professor and Head, Department of Transfusion Medicine, SCTIMST, Medical College P. O. Thiruvananthapuram - 6950I I, Kerala, India Email debasishgupta@gmail.com

Received: June II, 2019| Published: August 07, 2019
Abbreviations: RBC, red blood cells; CTT, conventional tube technique; CAT, column agglutination technology; IAT, indirect anti globulin test; DAT, direct anti-globulin test; HDFN, hemolytic disease of fetus and newborn

\section{Introduction}

The Rh blood group system consists of five important antigens $\mathrm{D}, \mathrm{C}, \mathrm{c}, \mathrm{E}$, and e. The D antigen is the most immunogenic of all the non-ABO antigens. Approximately $80 \%$ of individuals who are D-negative and exposed to a single D-positive unit will produce an anti-D antibody. ${ }^{1}$ Hemolytic disease of fetus and newborn (HDFN) occurs when there is a destruction of fetal/neonatal red blood cells (RBC) by the trans-placentally obtained IgG antibodies produced by the mother. The blocking of $\mathrm{D}$ antigen sites of RBC membrane of the fetus by the passively transferred IgG anti-D in cases of HDFN is called blocked- D phenomenon. The coating of maternal IgG type of anti-D prevents the agglutination of the Rh-(D) antigen positive red blood cells $(\mathrm{RBC})$ by the IgM D-antigen typing reagents. We are reporting two cases of Rh-(D) HDFN which were falsely typed as $\mathrm{Rh}$ (D) antigen negative with routine typing reagents and also had multiple allo-antibodies in the maternal serum.

\section{Materials and methods}

One EDTA and one clotted sample of the mother and the baby were obtained along with one EDTA sample of father and first child. Both the cases were referred from other hospitals. Blood group confirmation was done by forward and reverse grouping (Conventional tube Technique or CTT). Direct Anti globulin test of the baby and Indirect Anti globulin test of maternal serum were carried out using both Conventional tube technique and Gel cards-Column Agglutination Technology or CAT (Ortho Biovue system Anti-IgG-c3d; Polyspecific coomb's card). Antibody screening and antibody identification were performed by CAT (Biorad ID Diapanel-P). Red cell elution was performed by Gentle heat elution technique at $45^{\circ} \mathrm{C}$ for 10 minutes (Partial elution Technique). ${ }^{1}$ Eluted red cells were tested for D antigen with three different types of monoclonal anti D (IgG, IgM, IgG-IgM blend). DAT was performed on eluted RBC to confirm complete elution of coated antibodies and the cells were Rh phenotyped. Adsorption was performed by Enzyme-treatment of RBC's using 1\% Papain-cysteine. Antibody titration was done using Saline double dilution technique by CTT. Eluate was made to react with select cells by CAT (Ortho Biovue system Anti-IgG-c3d; Polyspecific coomb's card) for antibody identification and confirmation.

\section{Case history}

In the first case, a 27year-old lady G3P1L1A1 was admitted at 35 weeks of gestation. Her blood group was A-Rh- (D) negative and first child A-Rh-(D) positive. She received Anti-Rh-(D) prophylaxis after the first delivery and abortion. No history of any previous blood transfusions. Indirect Anti globulin Test (IAT) was positive with a titre of 64 at 16weeks of gestation. From 24weeks, fetal anemia warranted four intrauterine transfusions. Last transfusion was given five days prior to delivery. Baby was delivered at 35 weeks of gestation without 
any post-natal anemia or hyper bilirubinemia. Baby didn’t require any exchange or top-up transfusions after birth. Baby's blood group on cord blood sample was O-Rh-(D) negative. Being a case of known fetal anemia, cord blood samples of baby and maternal blood samples were sent to rule out Hemolytic Disease of Fetus and Newborn (HDFN). Blood group of mother was confirmed as A-Rh-(D) negative. IAT was positive with a titre of 512 and there was enhanced reaction at $1 \%$ papain-cysteine enzyme phase. Antibody screening and identification showed pattern of reactivity corresponding to "anti-D" and "anti-C" (Figures 1A\&1B). In order to rule out anti-G, maternal serum was adsorbed onto R2R2 ( $\mathrm{D}^{+} \mathrm{C}-$ ) cells. Eluate from these were cells tested for reactivity against r'r $\left(\mathrm{D}-\mathrm{C}^{+}\right)$cells, which came to be negative. Hence, anti-G was ruled out.

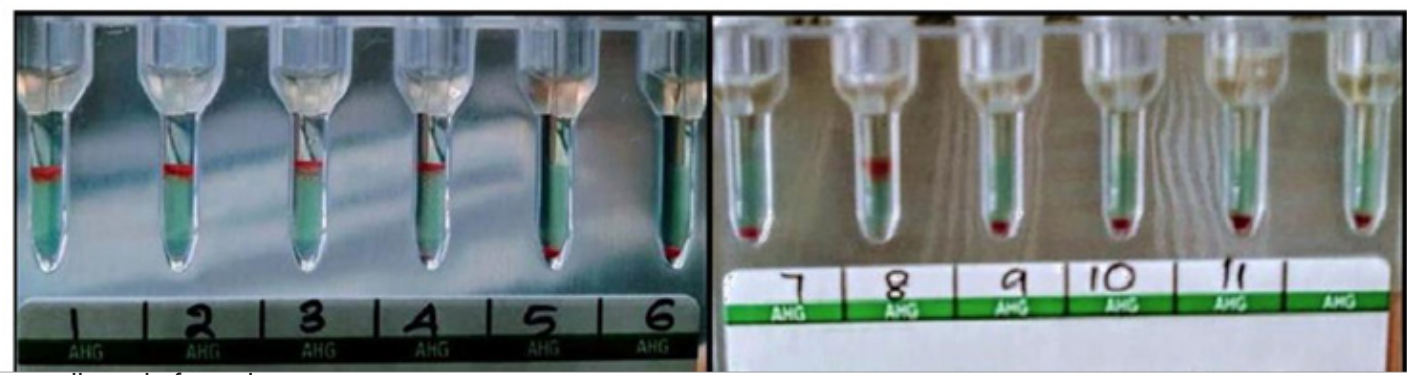

Figure I(A) Eleven cell panel of case I.
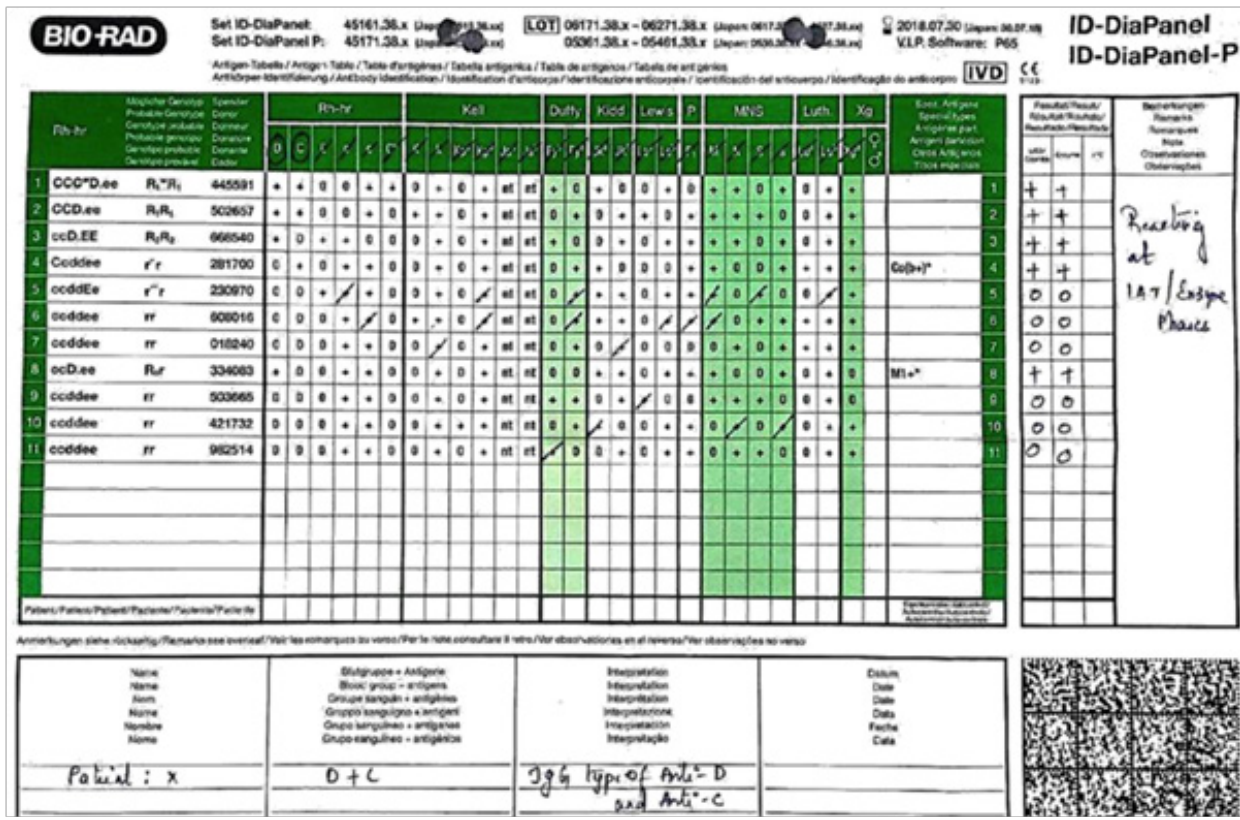

Figure I(B) Eleven cell panel anti gram of case I.

Maternal Rh extended phenotype was found to be D-C-E-c+e+t. Father and first child were both A Rh-(D) positive with Rh extended phenotype $\mathrm{D}+\mathrm{C}+\mathrm{E}-\mathrm{c}+\mathrm{e}+$.

The antibody titre against R2R2 (D+C-) cells were 512 and r'r $(\mathrm{D}-\mathrm{C}+)$ cells were 32 . Thus, anti-D titre was 512 and anti-C titre was 32.

Cord blood sample of baby was initially falsely typed as O-Rh(D) negative. Direct anti-globulin test showed weak mixed field reaction due to recent intrauterine transfusion with $\mathrm{O} R \mathrm{Rh}$-(D) negative red blood cells. Auto control of the baby was positive. Suspecting blocked-D phenomenon, baby's RBC's were subjected to gentle heat elution at $45^{\circ} \mathrm{C}$ which showed weak mixed field reaction with anti-D revealing baby to be Rh-(D) positive. Weak reaction could be explained due to the presence of donor RBC's from recent intrauterine transfusions. Even though baby was recently transfused extended phenol-typing of the eluted RBC's were performed to know the Rh(C)) antigenic status of the sample as mother had anti-C antibody. $\mathrm{Rh}-(\mathrm{C})$ antigen typing was negative. Eluate showed reactivity pattern of only anti-D. Hence, this was a case of Rh-HDFN presenting as blocked-D phenomenon due to trans-placentally obtained anti-D with coexistent maternal anti-C.

In the second case, a 32-year-old lady G2P1L1A0 was admitted at 37weeks of gestation for delivery following premature rupture of membrane. Her blood group was B-Rh-(D) negative and first child was B- Rh-(D) positive. She received Anti-Rh-(D) prophylaxis after first delivery. No history of previous blood transfusions or abortions.

Baby was delivered at 37weeks of gestation. Baby's blood group on cord blood sample was typed as B-Rh-(D) negative after birth. Because of postnatal hyper-bilirubinemia $(20 \mathrm{mg} / \mathrm{dL})$ and anemia $(9 \mathrm{~g} / \mathrm{dL})$ blood samples of the baby and mother were sent to rule out 
Hemolytic Disease of Fetus and Newborn (HDFN) on post-natal day.

Baby was given double surface phototherapy and bilirubin levels were gradually falling down. Baby didn't require exchange or top-up transfusions.

Blood group of mother was confirmed as B-Rh-(D) negative. IAT was positive with a titre of 32 and there was enhanced reaction at enzymatic phase. Antibody screening and identification panels showed pattern of reactivity corresponding to "anti-D", "anti- C" and "anti-E" (Figure 2A\&2B) . In order to rule out anti-G, maternal serum was adsorbed onto R2R2 (D+C-) cells. Eluate from these cells were tested for reactivity against r'r (D-+) cells and was found to be negative. Hence, anti-G was ruled out.
Maternal Rh extended phenotype was D-C-E-c+e+. Blood group of both father and first child was B Rh-(D) positive with extended $\mathrm{Rh}$ phenotype $\mathrm{D}+\mathbf{C}+\mathrm{E}+\mathrm{c}+\mathrm{e}+$ and $\mathrm{D}+\mathrm{C}+\mathrm{E}-\mathrm{c}+\mathrm{e}+$ respectively.

Cord blood sample of the baby was initially falsely typed as B-Rh(D) negative after birth. Sample was sent for further work up on postnatal day 2. Direct anti-globulin test and auto control were positive. $\mathrm{Rh}$ extended phenotype of the baby was $\mathrm{D}+\mathrm{C}+\mathrm{E}+\mathrm{c}+\mathrm{e}+$. Eluate showed reactivity pattern corresponding to anti-D and anti-C.

Hence, this was a case of HDFN presenting as blocked-D phenomenon due to transplacentally obtained anti-D and anti-C with coexisting anti-E.

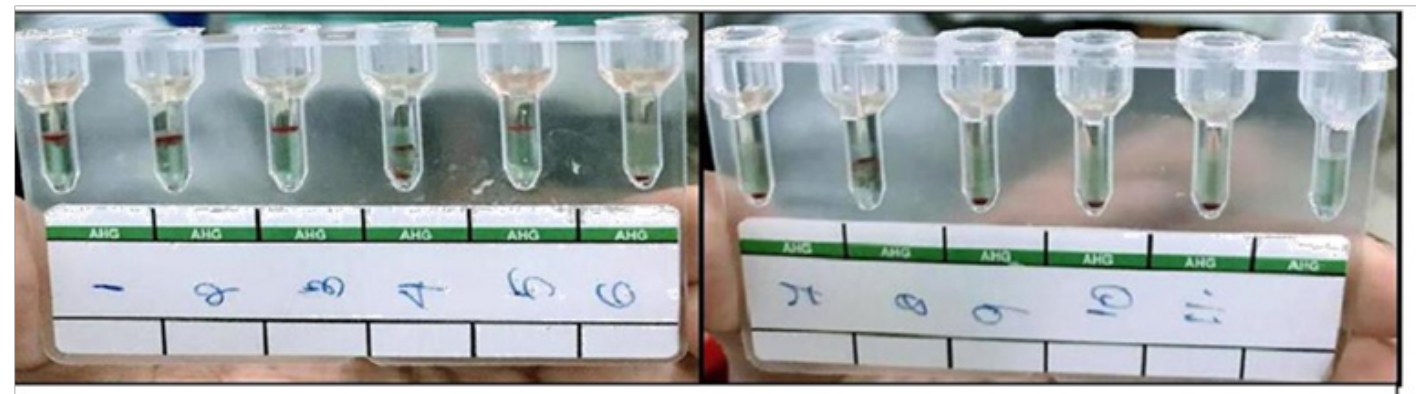

Figure 2(A) Eleven cell panel of case 2

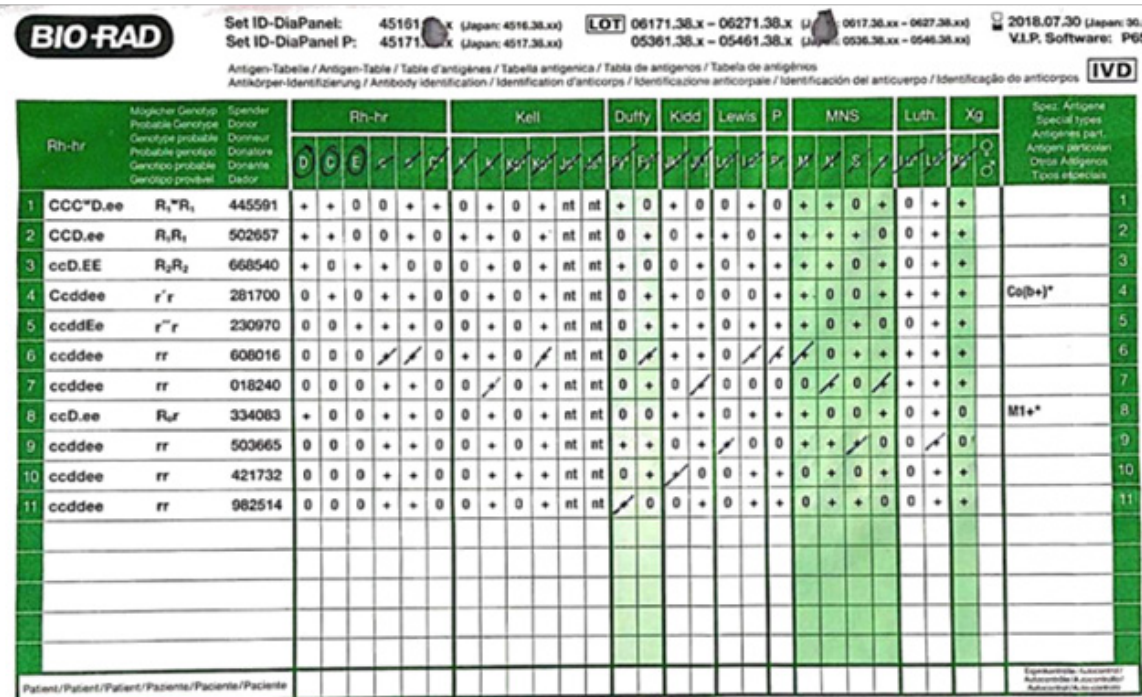

ID-DiaPanel IVD C ID-DiaPanel-P
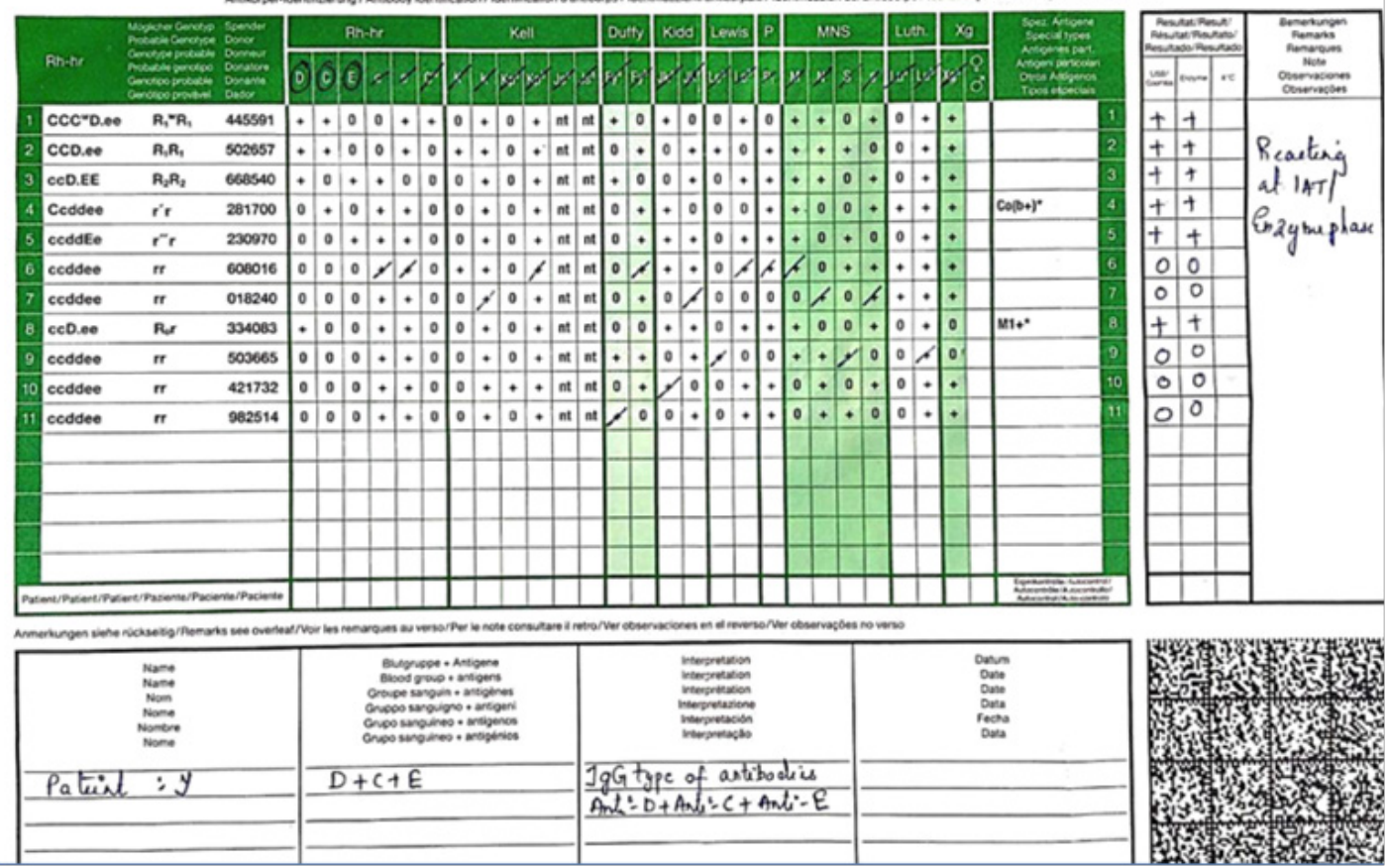

Figure 2(B) Eleven cell panel anti gram of case 2.

\section{Discussion}

Accurate Rh testing can be difficult if the red cells are heavily coated with IgG anti-D antibodies in a condition called blocked-D phenomenon. ${ }^{2}$ Nicolaides and colleagues in their study has showed the significant association between the degree of fetal anemia and maternal serum anti-D antibody concentration that is compatible with the pathophysiology of rhesus disease, which involves the extra-vascular destruction of fetal red cells that are coated with transplacentally derived maternal anti-D antibody. ${ }^{3}$

Sulochana and colleagues described a case of blocking anti-D with a maternal $\operatorname{IgG}$ anti-D titre of 32 , the $\operatorname{IgM}$ titre was 1,024 . In this case, the maternal and baby's RBC were initially grouped as B RhD 
negative in the local hospital. Due to profound jaundice and signs of kernicterus, the baby was transferred to the neonatology department of higher centre. Despite three exchange transfusions with B RhD negative blood the Direct Anti globulin Test (DAT) remained positive and free anti-D was still detectable in the baby's serum. Anti-D with a titre of 32 was eluted from the baby's RBCs. Antenatal grouping and atypical antibody screening had not been performed. ${ }^{2}$ This study shows that IgG anti-D need not be present in high titres to present with blocked D-phenomenon.

Verma and colleagues described a case of blocked $\mathrm{D}$ in $\mathrm{RhD}$ haemolytic disease of the fetus. At 20 weeks gestation, the maternal anti-D titre was found to be 256 by conventional tube technique. Subsequent ultrasound screening showed the fetus to be hydropic and percutaneous umbilical cord blood sampling confirmed fetal anemia ( $\mathrm{Hb} 5.4 \mathrm{~g} / \mathrm{dL}$ and hematocrit $13.9 \%$ ). The fetal $\mathrm{RBC}$ was grouped as $\mathrm{RhD}$ negative with a positive DAT, an eluate yielded anti-D, showing the $\mathrm{D}$ antigenic sites were blocked for the routine typing reagents to act. Successful intrauterine transfusion (IUT) was performed and post transfusion hemoglobin increased to $14.1 \mathrm{~g} / \mathrm{dL}$ and hematocrit $41.8 \%$. An icteric baby was delivered, again grouping as $\mathrm{RhD}$ negative, but on this occasion due to the O-Rh-(D) negative blood used for the IUT. ${ }^{4}$

Yohannes and colleagues describes a case report in which after several transfusions with D-negative blood, an $\mathrm{O}$ Rh-D negative woman was apparently sensitized to the $\mathrm{C}$ and $\mathrm{D}$ antigens. In her prenatal workup, it became evident that she had in fact not been sensitized to $\mathrm{D}$ or $\mathrm{C}$ antigen as such but to $\mathrm{G}$, which initially appeared as anti-D plus anti-C. Moreover, the fetus was affected significantly and was delivered at $32 \frac{1}{2}$ weeks with moderate hemolytic disease of the newborn. ${ }^{5}$ So, it is necessary to identify the presence of anti-G when the antibody identification shows reactivity pattern to both D and $\mathrm{C}$ antigens.

Hadley and colleagues in their study emphasizes the importance of continuous monitoring of $\mathrm{C}$-antigen positive fetuses who were genotyped as D-negative when maternal serum apparently contains anti-C+D; HDFN due to anti-G may be of life-threatening severity and so it would seem advisable in such cases to test for anti-G in maternal serum. ${ }^{6}$ In cases were only anti-G is present; mother has to be given routine anti-D prophylaxis to protect future D antigen positive fetuses. Palfi and colleagues in their study had proposed that the $G$ antigen seems to be highly immunogenic as anti-G occurred in 24 out of 27 patients compared to the occurrence of anti-D in 23 out of 27 patients in their study. ${ }^{7}$ Maley and colleagues mentions that cases where antibody identification reveals only anti- G, with or without anti-C, it is appropriate to administer anti-D immunoglobulin both during the pregnancy, and at the delivery of an $\mathrm{RhD}$ positive infant, to prevent the formation of anti-D.

Moran and colleagues documents the natural history of anti-E HDFN in pregnancy and shows that a substantial proportion of infants are sufficiently affected by anti-E and they do suffer from clinically important HDFN. Twenty-one percent of affected infants received exchange transfusion and $10 \%$ had severe or very severe disease. The single, very severely affected case had a maximum anti-E titre of $1 / 1$, highlighting the disparity between anti-E titre and disease severity. ${ }^{9}$

Markham and colleagues stated that the presence of multiple red blood cell antibodies is associated with increased odds for the development of significant HDFN. HDFN is more likely to occur in the presence of multiple red blood cell antibodies, especially in the presence of anti-(Rh) D. Heightened awareness of the increased potential for significant HDFN in the presence of multiple red blood cell antibodies may prove to be helpful to the clinician, permitting anticipation and more aggressive antenatal management of these patients. ${ }^{10}$

While interpreting the results of blood grouping on fetal or neonatal samples from an allo immunized pregnancy with potent antibody, the blocking phenomenon should be taken into account. A false negative $\mathrm{RhD}$ grouping can be seen when maternal IgG antibodies saturate all antigen sites on fetal red cells and leave no antigen sites for the anti -D reagent to attach with. A proper clinical history, including the history of any intrauterine transfusions and results of previous immunohaematological investigations done during the perinatal period, is also very important in order to reach the final diagnosis. ${ }^{4}$ All women, regardless of their D type, should be tested during each pregnancy for clinically significant unexpected serum antibodies, ideally at their first visit to the obstetrician. Anti globulin testing should be done with anti- IgG to detect preferentially those antibodies with the potential to cross the placenta and cause HDFN. ${ }^{11}$

Antibody identification in antenatal women are important for intrauterine transfusions also; since, always maternal allo-antibody need not be anti-D and it can be some other $\mathrm{Rh}$ and other minor blood group antibody which can complicate intrauterine transfusion. Usually O group Rh-(D) negative blood is preferred for intrauterine transfusion, but if the maternal antibody is other than anti-D and is against some other antigen present on the transfused $\mathrm{O}$ Rh-(D) negative red blood cells, then it will result in severe in-utero hemolytic disease of the fetus.

\section{Conclusion}

All cases of Rh-HDFN should not be merely considered to be due to anti-D. There can be coexistent other allo-antibodies contributing to it. Hence, proper identification of antibodies is as important as managing a case of HDFN to protect future pregnancies and to avoid the risk of mismatched transfusions.

\section{Acknowledgments}

We thank the Director, Sree Chitra Tirunal Institute for Medical Sciences and Technology and Department of Transfusion Medicine for permitting us to do the study. We also acknowledge Department of Pediatrics, SAT Hospital, Medical College, Trivandrum for providing blood samples for investigation.

\section{Conflicts of interest}

The author declares that there are no conflicts of interest.

\section{References}

1. Brecher ME. (American Association of Blood Banks) AABB Technical Manual. $15^{\text {th }}$ ed. Bethesda: AABB; 2008.

2. Sulochana PV, Mathai AJ, Sathyabhama S, et al. Blocked D phenomenon, a rare condition with $\mathrm{RhD}$ haemolytic disease of newborn - a case report. Int J Lab Hematol. 2008;30:244-247.

3. Nicolaides $\mathrm{KH}$, Rodeck $\mathrm{CH}$. Maternal serum anti-D antibody concentration and assessment of rhesus isoimmunisation. BMJ. 1992;304(6835):1155-1156.

4. Verma A, Sachan D, Bajpayye A, et al. RhD blocking phenomenon implicated for an immunohaematologic diagnostic dilemma in a case of RhD-haemolytic disease of fetus. Blood Transfus. 2013;11:140-142. 
5. Yohannes W, Akhter JE. Hemolytic Disease of the Newborn Due to Anti-C and Anti-G Masquerading as Anti-D. Am J Clin Pathol. 1985;84(6):769-72.

6. Hadle AG, Poole GD, Poole J, et al. Haemolytic Disease of the Newborn Due to Anti-G. Vox Sang. 1996;71:108-12.

7. Palfi M, Gunnarsson C. The frequency of anti-C + anti-G in the absence of anti-D in allo immunized pregnancies. Transfusion Medicine. 2001;11(3):207-210.

8. Maley M, Babb R, Chapman CE, et al. Identification and quantification of anti-D,-C and $-\mathrm{G}$ in allo immunized pregnant women. Transfusion Medicine. 2001;11:443-446.
9. Moran P, Robson SC, Reid MM. Anti-E in pregnancy. BJOG. 2000;107:1436-1438

10. Markham KB, Karen, Rossi BSN, et al. Hemolytic disease of the fetus and newborn due to multiple maternal antibodies. Am J Obstet Gynecol. 2015;213(1):68.e1-68.e5.

11. Judd JW. Practice guidelines for prenatal and perinatal immuno hematology, revisited. Transfusion. 2001;41(11):1445-1452. 